\title{
La infancia y la adolescencia como noticia en El Salvador
}

Recibido: 12/07/2013 - Aceptado: 08/09/2013

\section{Resumen}

Solamente el $7 \%$ de las noticias que se publican en la prensa, la radio y la televisión salvadoreñas se relacionan con la infancia, la adolescencia y la juventud. Este artículo se basa en una investigación realizada en 2012 y en la que se hizo un análisis de contenido a 292 textos informativos publicados entre junio y julio de ese año. El estudio establece que cuando en los medios se escribe de niños, niñas y adolescentes se hace únicamente de manera descriptiva sin profundizar en la temática abordada; no hay planteamientos positivos ni propositivos para solucionar las problemáticas; la información se escribe sin contexto; los textos informativos sobre este grupo poblacional no tienen mucha importancia dentro de los medios, pues solo un mínimo porcentaje de ellos aparece en las portadas o titulares. La investigación buscó analizar el tratamiento periodístico en los temas relacionados con la infancia y la adolescencia para conocer el panorama establecido por los medios de comunicación en esta temática.

\section{Palabras clave}

Noticias, medios de comunicación, periodismo, niñez, adolescencia, juventud.

\section{Camila Calles Minero}

\begin{abstract}
In El Salvador, only $7 \%$ of the news published in the press, radio and television are related to childhood, adolescence and youth. This article is based on research done in 2012 and in which we conducted a content analysis of 292 informative texts published between June and July of that year. The study states that when the media writes about children and adolescents is done cold, concrete, without delving into the problems, there are no positive proposals to solve the problem, the information is written without context. Informational texts about this topic do not have much importance in the media for only a small percentage of them appear on the covers or holders. This research was made by Universidad Tecnologica de El Salvador with financial support from Unicef. This article only presents a part of the descriptive results for the research conducted.
\end{abstract}

\section{Keywords}

News, media, freedom of information, journalism, childhood, adolescence, youth.

\section{Introducción}

“Mientras (los medios de comunicación) parecen hacer de termómetro, que registra un incremento de temperatura, en realidad forman parte del combustible con el que se alimenta la caldera."

Umberto Eco (1999)

Es innegable que el mundo está en plena revolución de la información, en donde los datos y el conocimiento cobran importancia, pues otorgan poder a quienes los poseen. Ya lo decía McLuhan (1996), que la información ha dejado de ser un instrumento para producir bienes económicos. Entre las líneas temáticas que circulan en las carreteras de la información están las relacionadas con niños, niñas y adolescentes, que se difunde a través de diversos vehículos, entre los que se encuentran los medios de comunicación.

Las nuevas sociedades, en medio de esa revolución del conocimiento y específicamente con la información sobre infancia y adolescencia, tienen que tomar en cuenta lo

Camila Calles Minero. Es Máster en Docencia e Investigación Educativa, Máster en Estudios Sociales de la Ciencia y la Tecnología, licenciada en Periodismo. Investigadora. Universidad Tecnológica de El Salvador. camila.calles@utec.edu.sv. 
dictado por la Convención sobre los derechos del niño (1989) en donde se reconoce que, para el pleno y armonioso desarrollo de su personalidad, niños, niñas y adolescentes deben crecer en el seno de la familia, en un ambiente de felicidad, amor y comprensión.

Además, se considera que "el niño debe estar plenamente preparado para una vida independiente en sociedad y ser educado en el espíritu de los ideales proclamados en la Carta de las Naciones Unidas y, en particular, en un espíritu de paz, dignidad, tolerancia, libertad, igualdad y solidaridad" (Acnur, 2012).

En ese sentido, los niños, las niñas y los adolescentes se tienen que ver, más que como promesas de futuro, como realidad presente, eso según instancias nacionales e internacionales, las que están utilizando ese enfoque para el trabajo en pro de los derechos de ese sector poblacional.

Teóricamente, y respondiendo a su deber ser, los medios de comunicación de masas reflejan la realidad, pero también la configuran. Si bien son un medio para conocer la realidad, en cierta manera constituyen un "espejo, que refleja la imagen de la sociedad respecto a sí misma. Son un filtro que selecciona partes de la experiencia para dedicarles una atención especial y descarta otros aspectos, son una señal que indica activamente el camino, orienta, instruye" (McQuail, 1996).

Esa construcción de la realidad salvadoreña que realizan la prensa escrita, la radio y la televisión es importante de analizar, desde el hecho que la infancia está representada por estereotipos culturales que erosionan la imagen de niños, niñas y adolescentes, según Unicef (2011). Estos estereotipos rondan entre la participación de este sector poblacional en actos delincuenciales; se presentan como víctimas e indefensos, entre otros.

El trabajo periodístico, en todo el mundo, responde a los entornos sociales en los que se desarrolla. En el caso de El Salvador, es evidente que la violencia es un tema cotidiano y ocupa la mayor parte de espacios en los medios de comunicación, en sus diferentes formatos. El 78 \% de las franjas infantiles de televisión que se transmiten en el país tienen al menos una acción de violencia (Calles, 2011). Es necesario inspeccionar si los productos periodísticos incluyen violencia en contra de la infancia salvadoreña.

Los medios son una fuente de confianza por parte de la población. Esta afirmación la ratifica los resultados de la LVI encuesta del Centro de Investigación de la Opinión Pública Salvadoreña (Ciops), de la Universidad Tecnológica de El
Salvador, correspondiente al mes de enero de 2012, y en la cual se registra que la población salvadoreña le coloca una nota de 7.5 a los medios de comunicación.

Muchos teóricos se han encargado de establecer que los mass media establecen pautas de comportamiento en sus adeptos. Desde inicios del siglo $\mathrm{XX}$, los efectos que producen a sus consumidores forman parte de objeto de estudio y preocupaciones de investigadores de la materia. Desde entonces se plantea el establecer parámetros para entender qué provoca, en una persona, el ver la televisión, leer el periódico o escuchar la radio; hoy se suma el uso de internet.

Evenland (2003) asegura que los efectos que producen los mass media es uno de los grandes paradigmas de la comunicación de masas y en la que se enmarcan muchos estudios. Por su parte, Alonso (2010) explica que existen diferentes teorías acerca del estudio de los medios de comunicación y sus efectos. Desde su surgimiento, estos medios se constituyeron como elemento importante en la estructura de una sociedad, en cuanto a que son los transmisores de valores, símbolos, comportamientos, ideologías, formas de enfrentar la vida, elementos de la realidad, entre otras cosas.

En ese sentido, Castillo (2011) dice que disponer de los medios de comunicación significa, en la actualidad, poseer un poder social no tanto por el contenido que transmiten, sino por el ambiente que crea, la atención y el mimetismo que despierta. El medio actúa como un espejo que refleja la realidad social $y$, al mismo tiempo, es el lugar en el que se crea esta.

Gutiérrez Vidrio (2010) asegura que los medios masivos de comunicación desempeñan un papel central en la configuración de la cultura moderna de las sociedades, ya que son los mediadores necesarios y omnipresentes entre el acontecer social y cada persona que consume los contenidos mediáticos. Esta autora señala que una característica del discurso de los medios de comunicación es que estos construyen una representación de la realidad a la que aluden. Desde una perspectiva construccionista, la realidad no existe como tal, sino que es socialmente construida; en esa construcción, los medios tienen participación directa.

La realidad a la que se refieren los medios de comunicación "es compleja, diversa y cambiante; es una realidad social poliédrica de la que sólo damos cuenta de algunas de sus caras" (Rodrigo, 2005, citada por Gutiérrez Vidrio, 2010, p. 23). 
Con relación al trabajo periodístico, Unicef (2005) establece que los periodistas tienen el poder y la responsabilidad, desde su tarea cotidiana, de contribuir a la modificación de estereotipos e imágenes negativas hacia representaciones que permitan ver a los niños, las niñas y los adolescentes como ciudadanos con derechos y como actores sociales valiosos y relevantes en el desarrollo de sus sociedades; más que como promesas del futuro, como realidad del presente.

El trabajo periodístico se muestra a los usuarios de la información a través de diversos géneros, los cuales es necesario diferenciar para determinar sus características discursivas, así como su función y estructura. Existen diversas; una de las clásicas es la planteada por Martínez Albertos (1988), quien los clasifica en informativos, interpretativos e híbridos.

Martín Vivaldi (1981), Santamaría (1990) y Martínez Albertos (2002) coinciden en que existen tres tipos de géneros periodísticos: Ios de opinión, en donde están los editoriales, artículos, columnas, comentarios; los informativos, que son la noticia, reportajes, entrevistas; y los interpretativos, en donde la diferencia básica con los informativos es el grado de análisis de los hechos.

En cuanto a las temáticas que se publican en los medios de comunicación, autores como Grillo (2001) aseguran que en los textos informativos, ya sea radio, prensa, televisión e internet sobresalen las noticias con enfoques negativos. Sin embargo, esta postura la estableció desde 1969 McLuhan, quien dijo que "las auténticas noticias son las malas noticias; como la prensa vive de la publicidad, y la publicidad está formada por buenas noticias, se necesita gran cantidad de malas noticias para vender las buenas. Incluso la buena noticia del evangelio puede venderse únicamente gracias al fuego del infierno" (McLuhan 1969).

Eco (1999) establece que los medios de comunicación "mientras parecen hacer de termómetro que registra un incremento de temperatura, en realidad forman parte del combustible con el que se alimenta la caldera". En ese sentido, la imagen de infantes y adolescentes en El Salvador es dibujada también por los contenidos mediáticos que muestran el mundo de la infancia y la adolescencia con la utilización de determinado lenguaje, con la divulgación de temas específicos, consultando ciertas fuentes, publicando con géneros determinados.

Al hablar concretamente de la infancia y la adolescencia, en el año 2007, el World Youth Report, de la Organización de las Naciones Unidas, estableció que, a pesar de que la juventud representa al $18 \%$ de la población mundial (1.2 millones de personas con edades entre los 15 y 24 años) y que es esencial para el desarrollo de las sociedades, además cuentan con un nivel de educación mayor que las generaciones anteriores a ellos y constituyen el $25 \%$ de la población laboralmente activa, es un sector poblacional que sigue afectado por la pobreza y las limitaciones culturales y sociales (Flacso, 2011).

En El Salvador, el $40 \%$ de sus habitantes son menores de 18 años, es decir, 2.4 millones de salvadoreños son niñas, niños y adolescentes, según datos de Unicef (2011) y de la Dirección General de Estadística y Censos de El Salvador (Digestyc, 2007).

Según Unicef (2007), para el 2007 se destinaban $\$ 3.4$ de $\$ 100$ del producto interno bruto (PIB) para proyectos en beneficio de la niñez salvadoreña. El $17 \%$ del presupuesto general de la nación va para la niñez. Este organismo internacional asegura que anualmente el Estado salvadoreño gasta $\$ 317$ por cada niña, niño y adolescente. En cuanto a los gobiernos locales, estos invierten $\$ 2.80$ anual por un menor de 18 años.

Por su parte, Flacso (2011) asegura que la juventud de Latinoamérica sufre las consecuencias de estilos de desarrollo excluyentes, puesto que existen brechas en cuanto a la formación de capacidades y oportunidades, y porque los jóvenes no comparten las mismas metas, beneficios ni el mismo futuro, pese a vivir en un mismo territorio geográfico o sociedad.

Los problemas que afectan a los adolescentes salvadoreños son predominantemente los de tipo emocional y se asocian a una falta de valores morales, desmotivación para el estudio y la ausencia del sentido de la vida (Compañía de Jesús, 2006; citado por Flacso, 2011).

Merlo (2002) asegura que son muchos los niños y jóvenes de nuestra sociedad que tienen altos niveles de miedo ante el mundo. ¿En qué se pueden transformar a corto y mediano plazo? Paradójicamente, los adolescentes terminan transformándose en una población de riesgo generadora de violencia social.

En la actualidad, y a escala mundial, cobra cada vez más importancia el enfoque del niño, la niña y el adolescente como sujetos de derecho, el cual, según Unicef (2005), inicia con la Convención Internacional sobre los Derechos del Niño, adoptada por la Asamblea General de las Naciones Unidas en 1989, y en la que se concibe a la infancia y la adolescencia como personas que, por ser tales, gozan del 
atributo inherente de los derechos humanos en cuanto que son sujetos sociales y protagonistas.

Unicef (2011) asegura que este enfoque suplantó al que veía a este sector de la población más bien como objeto de protección solo en tanto que es excluido social. Sin embargo, hoy en día se sostiene que la niñez no es sujeto titular de derecho, sino eje de necesidades.

Es relevante que se visualicen temáticas que pueden aludir a los derechos de la infancia y la adolescencia, y con ello promover su respeto y denunciar su vulneración. Es importante tomar en cuenta que los medios de comunicación social son agentes de socialización, junto con la familia, la escuela, la iglesia y los grupos de referencia social. McLuhan (1996) estableció que todos los medios son metáforas activas por su poder de traducir la experiencia en nuevas formas.

Los medios de comunicación tienen una influencia innegable en la manera en que percibimos el mundo. De ahí la importancia en relación con la construcción de los contenidos que se transmiten en ellos, así como en la forma en que se dan a conocer.

\section{Metodología}

La población que se investigó está conformada por siete noticieros de televisión, siete de radio y tres periódicos; todos de cobertura nacional. Los noticieros televisivos son parte de la programación de canales de señal abierta salvadoreña, las radios pertenecen al espectro de frecuencia modular; y en el caso de los periódicos, son de circulación nacional. Las entregas noticiosas de radio y televisión analizadas son parte de los horarios estelares. En cuanto a los periódicos, se vieron todos los ejemplares diariamente. El monitoreo se realizó en los meses de junio y julio de 2012.

Las diecisiete producciones noticiosas (siete de televisión, siete de radio y tres de prensa impresa) sumaron 3.703 textos periodísticos, de las cuales 292 se refirieron a niños, niñas y adolescentes; y a las que se les aplicó un análisis de contenido basado en un instrumento diseñado con las variables que se debían buscar y que fue aplicado a cada información sobre temas de infancia y adolescencia.

\section{Resultados}

El análisis de textos periodísticos publicados en entregas noticiosas de prensa, radio y televisión consistió en buscar variables, entre ellas: lugar de la publicación, extensión de la noticia, aparición o no en titulares y portada, fuentes consultadas, géneros periodísticos utilizados, presencia o no de contexto en la información, presencia o no de propuestas positivas y propositivas en el texto. A dichas variables se les aplicó la prueba estadística de independencia Chi-cuadrado de Pearson, con el objetivo de comparar variables referidas al abordaje periodístico y el tipo de medio de comunicación (prensa, radio o televisión).

En total se recolectaron 3.703 textos informativos publicados durante los meses de junio y julio en diecisiete medios de comunicación salvadoreños (siete radios, siete canales de televisión y tres periódicos). De ese total de productos periodísticos, 292 corresponden a la temática de niñez y adolescencia.

De los productos periodísticos publicados y referidos al tema de infancia y adolescencia, la mayor parte pertenece a la prensa, con un total de 173 informaciones. En cambio, la radio tiene 61 textos informativos y la televisión 58.

Las 292 informaciones publicadas en los tres tipos de medios de comunicación analizados tienen un lugar físico determinado y la extensión en cada uno de los medios. El análisis estadístico estableció que, en el caso de la prensa escrita, de los 173 textos periodísticos publicados y relacionados con el tema de infancia y adolescencia, 125 de ellos tienen una extensión de uno a cinco párrafos; catorce de ellos, de seis a diez párrafos; y 34 tienen más de once párrafos. En el caso de la radio y la televisión, el análisis establece que, de los 119 productos periodísticos que suman entre estos dos medios de comunicación, 102 tienen una duración de uno a dos minutos, y diecisiete duran de tres a más minutos.

La importancia que los medios de comunicación otorgan a los textos periodísticos queda evidente en la ubicación en la publicación. El análisis estableció que de las 292 informaciones sobre infancia y adolescencia publicadas durante junio y julio de 2012, aparecen 200 al inicio del periódico o de los noticieros radiales o televisivos; 59 fueron publicadas al final de la publicación y 33 se divulgaron al inicio.

Las informaciones cobran importancia en los medios de comunicación en la medida en que aparecen o no en las portadas y titulares de las entregas noticiosas diarias. El análisis estableció que, de las 292 publicaciones, solo el $12 \%$ aparece en las portadas; esto significa que 38 productos periodísticos fueron parte de las portadas o titulares; en cambio, 254 no aparecieron en ellos. Se puede apreciar el detalle por medio de las informaciones publicadas en portadas o titulares en la figura 1. 


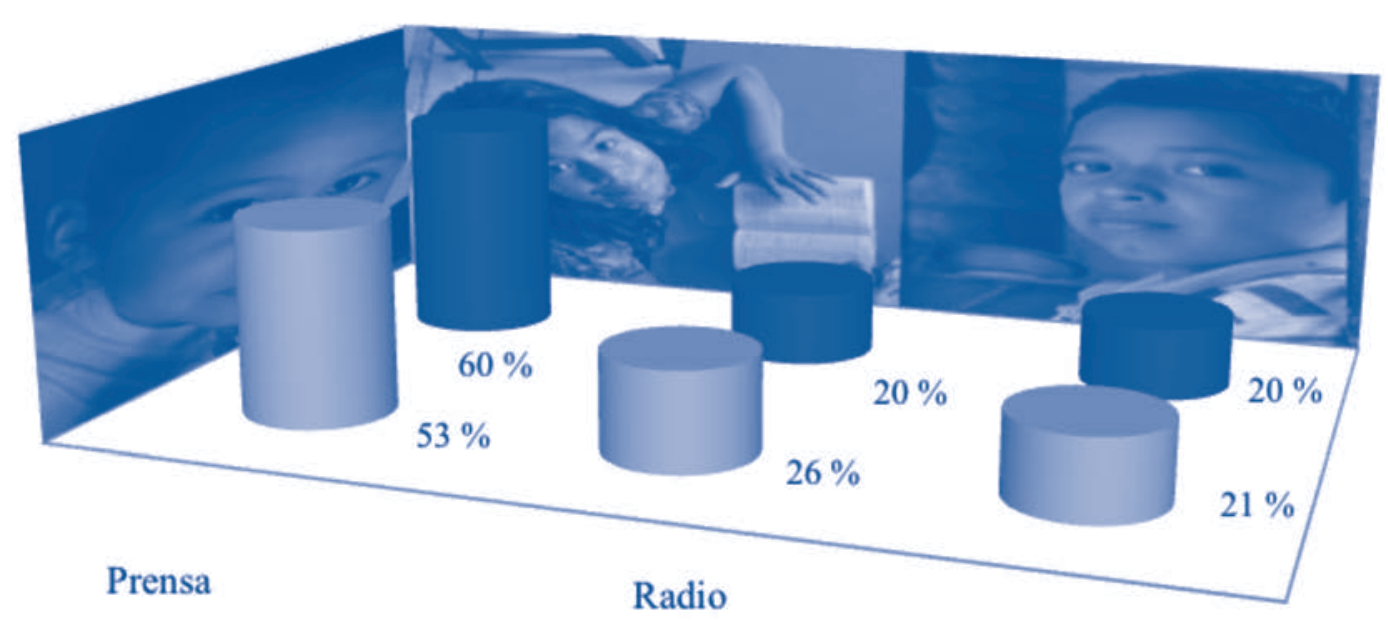

Televisión

\section{Figura 1. Información de infancia y adolescencia que aparece en las portadas y titulares}

Los temas referidos a la infancia y la adolescencia que abordan los medios, y que fueron inspeccionados como parte del estudio, fueron los siguientes: actividades de asistencia y solidaridad; actividades sociales, deportivas, entretenimiento; conflicto con la ley, cultura, derechos de la niñez y la adolescencia, discriminación, educación, familia, Lepina, maltrato; migración, muerte natural, muerte por violencia; pobreza, salud y trabajo infantil. En la figura 6 se observa la distribución de la ubicación dentro del medio.

Las cinco temáticas que más aparecen en la prensa, radio y televisión son las referidas a muerte por violencia, que representa el $16,3 \%$ de los textos publicados. En segundo lugar están los conflictos con la ley, que significan el 12,9\%; en tercer lugar aparecen los textos informativos referidos a la violencia, con el 10,9\%; en cuarto lugar está la temática de educación, con el 10,5 \% de los textos; y en quinto lugar están los textos de salud, con el 10,2\%.

Cuando se compara el tema del que trata el producto periodístico con la ubicación dentro del medio, la prueba no paramétrica del Chi-cuadro $\left(X^{2} 6=34.627 ; p=.344\right)$ establece que no hay diferencia estadísticamente significativa entre esas dos variables. En la figura 3 se puede observar que las temáticas que más se tratan son las que aparecen con mayor frecuencia a la mitad de la entrega noticiosa. 


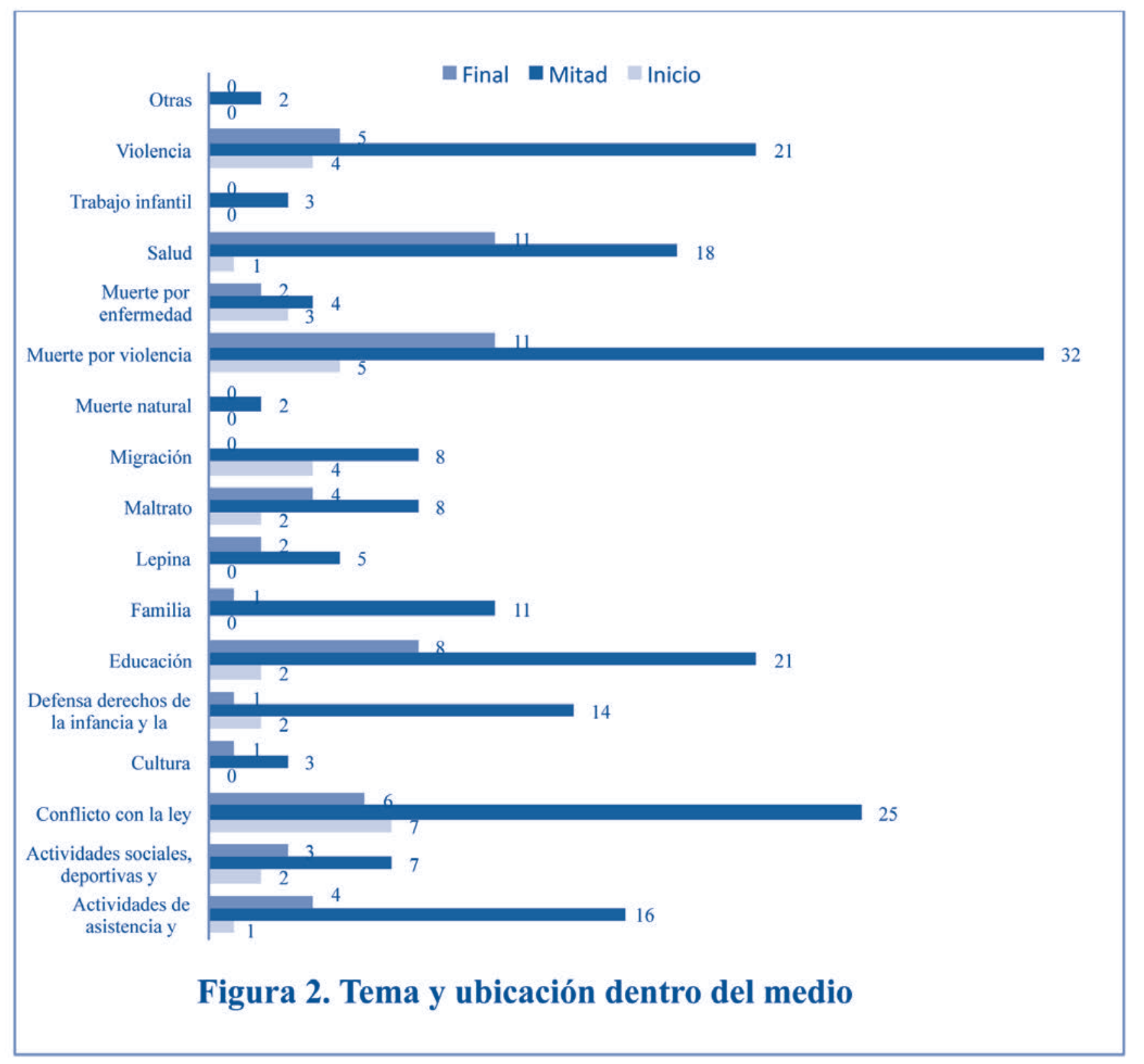


Como parte del tratamiento de los textos informativos, se analizó si El producto periodístico tiene o no contexto, se determinó que, de las 292 publicaciones en los medios de comunicación salvadoreños sobre el tema de infancia y adolescencia, el $43 \%$ (154) no tiene contexto y el $47 \%$ (138) sí lo tiene.

Al buscar la relación entre si El producto periodístico tiene o no contexto y el Tipo de medio de comunicación, la prueba de Chi-cuadrado $\left(\mathrm{X}^{2} \mathrm{6}=28.190 ; \mathrm{p}=.000\right)$ indicó que sí existe diferencia significativa entre el Tipo de medio de comunicación y el hecho de que El producto periodístico tiene o no contexto.

El $75 \%$ de los textos publicados y que tienen contexto (138) corresponde a Prensa; el $25 \%$ restante está dividido entre $13 \%$ de informaciones radiales y el $12 \%$ de televisivos. En el caso de los contenidos informativos que no tienen contexto (154), el $45 \%$ corresponde a Prensa, el $28 \%$ a Radio y el 27 \% a Televisión. Esto significa que radio y televisión son los medios que menos publican contexto en sus contenidos informativos.

Otra variable que se tomó en cuenta para analizar el tratamiento del tema de la infancia y la adolescencia en los medios de comunicación salvadoreños fue el uso de las fuentes informativas. Se buscó la presencia de las siguientes posibles fuentes de información: asociaciones civiles, asamblea legislativa (diputados), Fiscalía General de la República, FGR, infantes y/o adolescentes, jueces, ministerios, ONG, organismo internacional, presidencia de la República, Policía Nacional Civil, PNC, y otras.

Se encontró que la fuente que más presencia tiene en los textos periodísticos es la PNC, con el 27,2 \%; seguida de los diferentes ministerios públicos, que representan el $24,5 \%$. En tercer lugar de frecuencia está la utilización de otras fuentes (familiares, vecinos), que significan el 19,4 \% de las fuentes consultadas, para la construcción de los textos periodísticos. La voz de infantes y adolescentes está en el $10,8 \%$ de los textos publicados.

Al correr la prueba de interdependencia Chi-cuadrado ( $\mathrm{X}^{2}$ $6=25.795 ; p=.057)$ para verificar la relación entre el Tipo de fuente consultada y el Tipo de medio de comunicación, se estableció que no hay diferencia significativa entre esas variables, por lo que, independientemente del medio, se utiliza a la PNC como principal fuente de información en los textos referidos al tema de la infancia y la adolescencia (ver figura 3).

En el uso de las fuentes se denota que, para el caso de la Asamblea Legislativa, el medio que más hace uso de ella es la radio. Este mismo medio no utiliza a los organismos internacionales como fuente de información, al menos en los textos monitoreados en junio y julio de 2012.

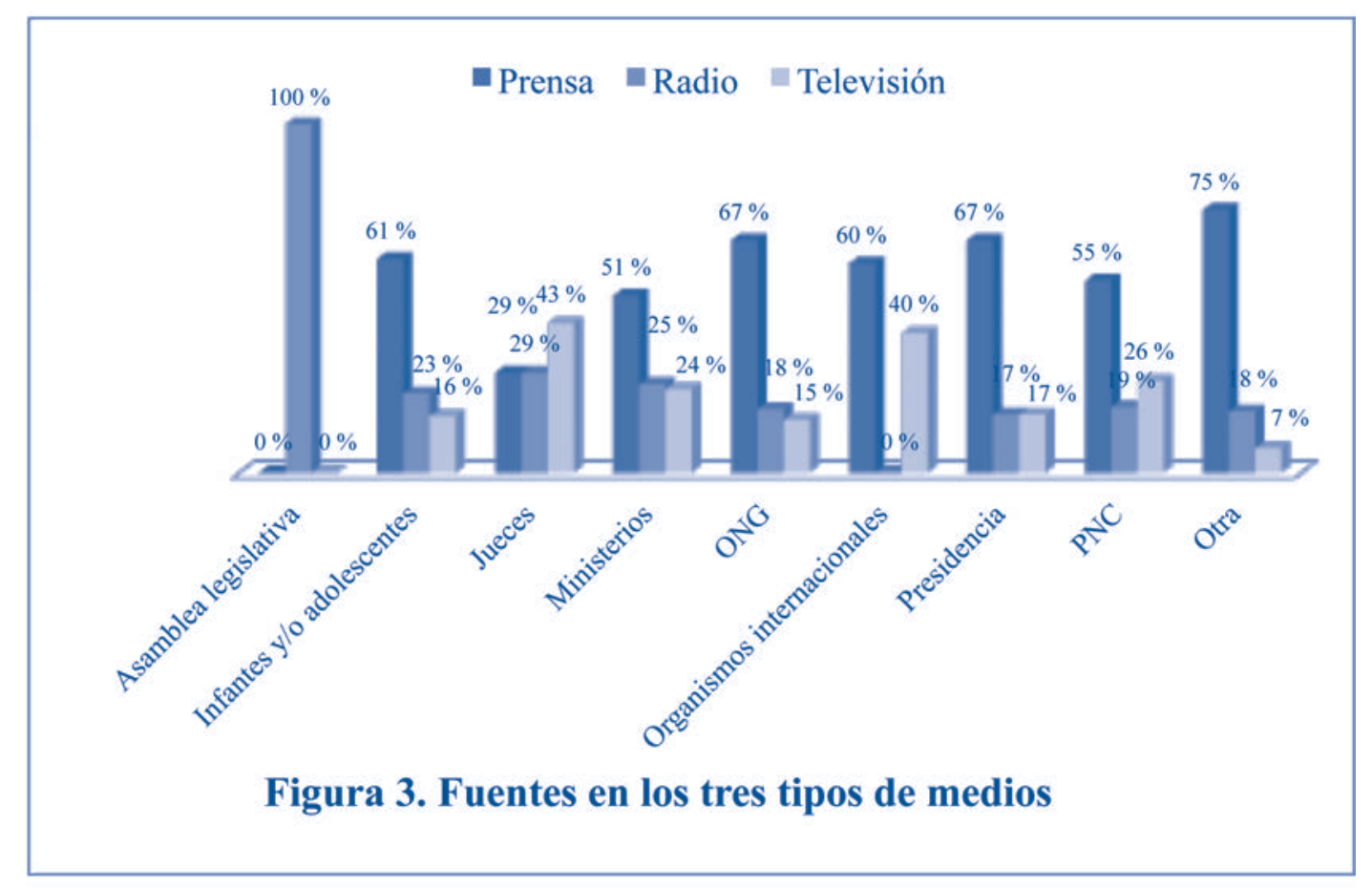


Siempre en relación con las fuentes informativas, se verificó la cantidad de fuentes utilizadas para la construcción de los contenidos informativos relacionados con la infancia y la adolescencia. Se encontró que prensa, radio y televisión utilizan con mayor frecuencia dos fuentes. La prensa escrita es el medio que tiende a utilizar más fuentes, en relación con la radio y la televisión.

La prueba de Chi-cuadrado $\left(X^{2} 6=32.014 ; p=.000\right)$ indicó que entre las variables Tipo de medio de comunicación y Número de fuentes consultadas existe una diferencia estadísticamente significativa. Eso quiere decir que depende del medio para la utilización de la cantidad de fuentes para construir el texto periodístico relacionado con el tema de la infancia y la adolescencia.
En cuanto a las fuentes consultadas, de acuerdo con la temática que se trata en las informaciones referidas a la infancia y la adolescencia, y al analizar únicamente las cinco temáticas más publicadas, se tiene que la PNC aparece como la entidad más consultada, tanto en el tema de conflictos con la ley como en las muertes por violencia. En los temas de educación y salud, la fuente más consultada son los ministerios. Los infantes y los adolescentes aparecen como fuente de información, con mayor frecuencia en los temas relacionados con la educación y la salud. En los temas de violencia, vista esta de manera general, las fuentes que aparecen con mayor frecuencia son las ONG y otras fuentes, como familiares de los infantes y los adolescentes y la población en general (ver figura 4).

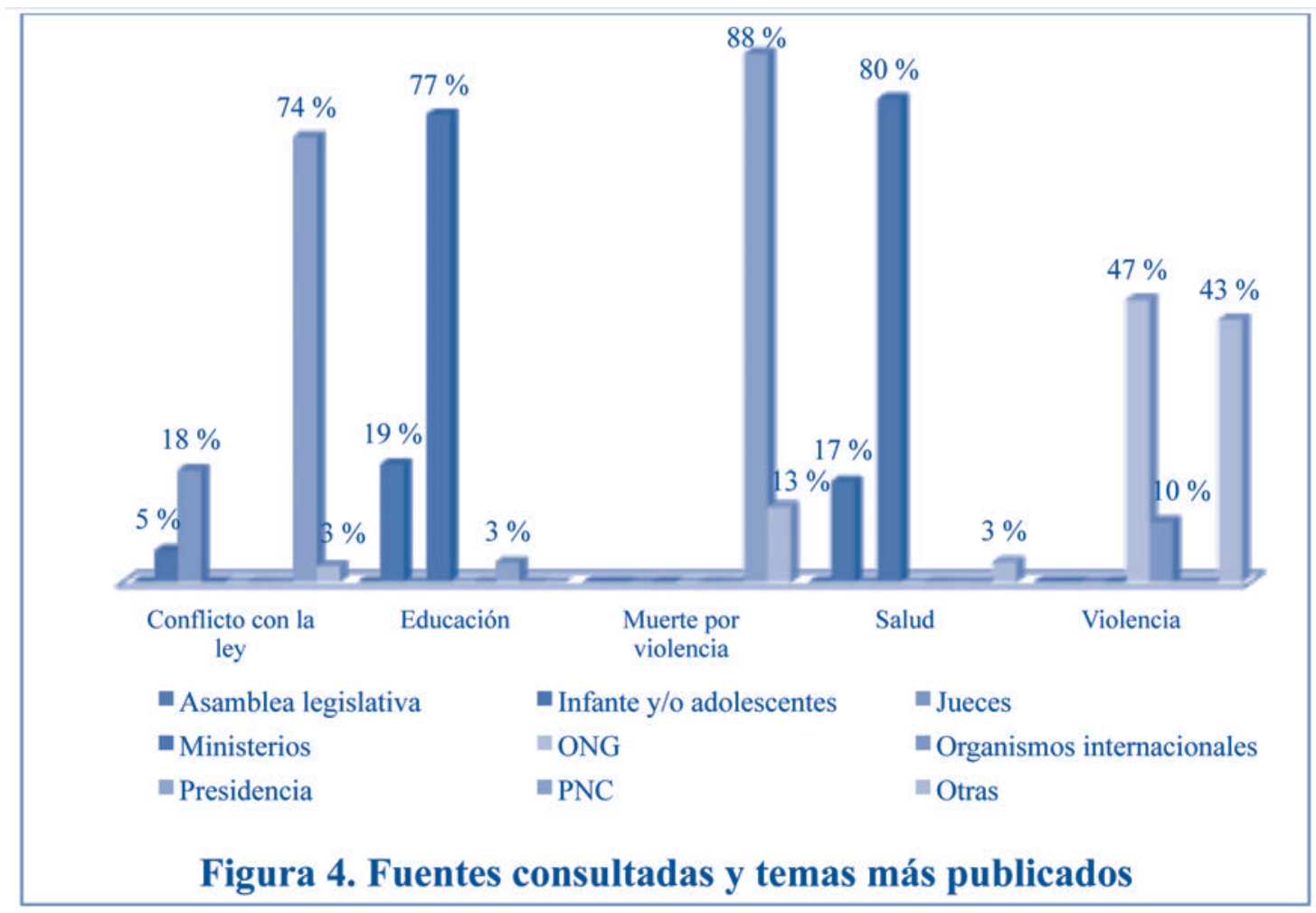

En cuanto al número de fuentes informativas utilizadas para la construcción de los contenidos informativos con mayor frecuencia de publicación, el estudio estableció que los temas de Muerte por violencia (60 \%) y Salud (53 \%) utilizan dos fuentes con mayor frecuencia. En el tema de violencia (50\%), se utiliza la mayoría de veces solo una fuente. En el caso del tema de Conflicto con la ley, el uso de una fuente (42\%) no varía en nada con el uso de dos fuentes (42\%).
El estudio también indagó sobre la presencia o no de planteamientos propositivos en los textos informativos relacionados con la infancia y la adolescencia, ante lo cual se encontró que, en el 74,4 \% de las informaciones, no existen ideas de nuevas y positivas propuestas ante los problemas que tratan (ver figura 5). 


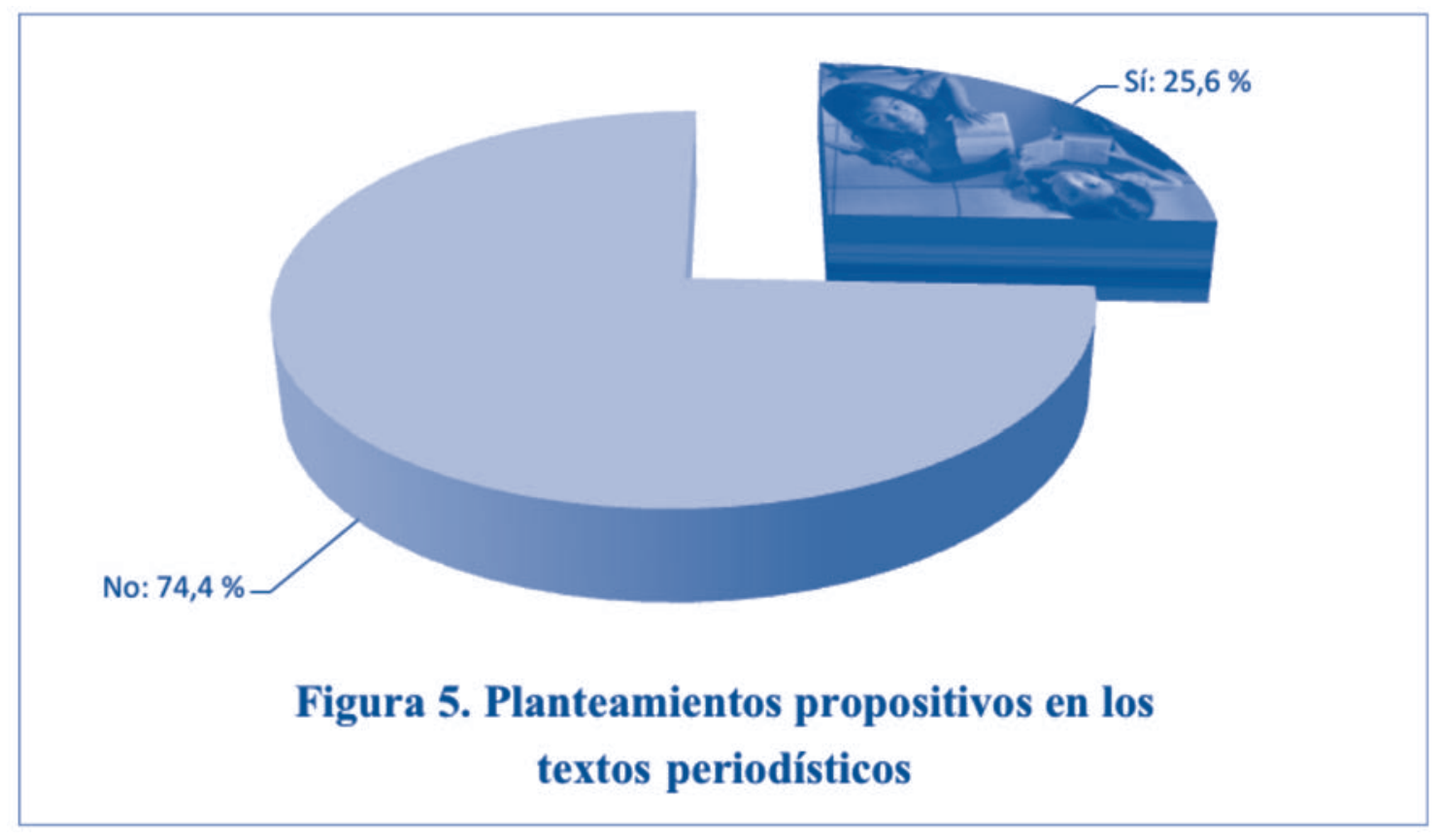

Al relacionar las variables de Planteamientos propositivos y/o positivos en torno al tema referido en los productos periodísticos con el Tipo de medio de comunicación, la prueba Chi-cuadrado $\left(X^{2} 6=10.232 ; p=.006\right)$ indicó que existe diferencia estadísticamente significativa entre ellas. Es decir, que no todos los medios de comunicación analizados tienen igual presencia de propuestas positivas.

Prensa es el medio que más utiliza este recurso periodístico a la hora de abordar la temática de la infancia y la adolescencia. El $73 \%$ de las informaciones publicadas y que tienen propuestas aparecieron en Prensa; el $19 \%$ en Radio y el $8 \%$ en Televisión. En cuanto a las informaciones sin planteamientos propositivos, el $55 \%$ apareció en Prensa, el 23 \% en Televisión y el 22 \% en Radio.

\section{Conclusiones}

- La prensa, la radio y la televisión escriben poco sobre la infancia y la adolescencia, por lo que se deduce que los medios de comunicación salvadoreña prefieren dedicar espacios para otros temas que, de acuerdo con la filosofía del medio, son más relevantes.

- Cuando se escribe de temas relacionados con la infancia y la adolescencia, los medios de comunicación no dan la relevancia que merecen. Eso se establece de acuerdo con la ubicación dentro de las publicaciones, puesto que muy pocos aparecen en las portadas y en los titulares. Son temas de las páginas interiores y no siempre tienen páginas completas.
- Los temas que más se publican tienen relación con el contexto de violencia que vive el país. Esto denota que el tema más relevante dentro de la dinámica periodística salvadoreña es la violencia. Esto compagina con las cifras de actos violentos a escala nacional. Los medios entonces replican su entorno.

- Las fuentes que utilizan los periodistas para construir las noticias relacionadas con la infancia y la adolescencia son oficiales; muy poco aparece la voz de los infantes y adolescentes, incluso en temas relacionados directamente con ellos, como actividades concretas sobre la infancia y la adolescencia.

- Los medios de comunicación transmiten mensajes con pocas fuentes; los temas son muy poco variados, hace falta contexto y propuestas en las informaciones; la profundidad de los textos se queda corta al solo publicar noticias, dejando a un lado géneros como el reportaje y la crónica, que podrían dar más elementos de análisis.

\section{Recomendaciones}

- Con esta investigación queda abierta la puerta para la realización de otros estudios en los que se pueden abordar las consecuencias en las niñas, los niños y los adolescentes de vivir bajo determinados estigmas, en donde se refuerzan estereotipos culturales como la discriminación. Los mass media establecen pautas de comportamiento en sus adeptos. Desde inicios del siglo $X X$, los efectos que producen los medios de comunicación a sus consumidores forman parte de 
investigaciones y preocupaciones de estudiosos de la materia. Desde entonces se plantea el establecer parámetros para entender qué provoca, en una persona, el ver la televisión, leer el periódico o escuchar la radio. Hoy en día se suma el uso de internet.

- Con los datos surgidos de este estudio se pueden hacer más investigaciones desde elárea de las comunicaciones, en cuanto al conocimiento o no de los derechos de la infancia por parte de los periodistas; analizar la puesta en marcha de leyes como la Lepina y cómo se habla de ella en los medios de comunicación.

- Se sugiere que este estudio de contenido realizado se vuelva a realizar en un año para poder determinar avances o no en los temas referidos a la infancia y adolescencia, sobre todo porque existen programas de capacitación a periodistas con relación al enfoque de derecho. Dado que existen estudios de este tipo en Latinoamérica, se propone hacer un análisis comparativo, en este sentido, de El Salvador y otros países de la región.

- Los hallazgos brindados por este estudio dio se deben socializar al interior del gremio periodístico salvadoreño, puesto que, más que señalar errores, lo que se pretende es mostrar realidades para contribuir en la construcción de una mejor sociedad.

- Ya que existen manuales periodísticos sobre cómo escribir sobre infancia y adolescencia, se sugiere que, con los hallazgos del estudio, se diseñe un manual propio para periodistas en El Salvador, tomando en cuenta la realidad peculiar del país, para que se interiorice en los medios de comunicación. Este manual debe de elaborarse con la participación de periodistas en ejercicio de los diversos medios.

\section{Referencias}

Acnur (2012), Convención de los derechos del niño. Recuperado de http://www.acnur.org/t3/fileadmin/ scripts/doc. php?file=biblioteca/pdf/0021

Alonso (2010), La evolución de la teoría de los efectos de los medios de comunicación de masas: la teoría de la espiral del silencio a partir de la construcción de la realidad social por parte de los medios de comunicación de masas. Universitat Pompeu Fabra. España.

Castillo (2011), Los medios de comunicación como actores sociales y políticos. Poder, Medios de Comunicación y Sociedad. Libros Básicos en la Historia del Campo
Iberoamericano de Estudios en Comunicación, número 75 , febrero-abril.

Convención sobre los derechos del niño (1989). Unicef: El Salvador.

Digestyc (2007), Censo de población y vivienda. El Salvador. Eco, U. (1999), La estrategia de la ilusión, 3era. edición. España: Lumen.

Eveland, W. (2003), A 'mix of attributes' approach to the study of media effects and new communication technologies. Journal of Communication, 53, 3, 2003. Pp. 395-410.

Flacso (2011), Una mirada a la juventud. Contextos, condiciones y desafíos en Guatemala, El Salvador y Nicaragua. El Salvador: Talleres Impresos Quijano.

Grijelmo, A. (2002), El estilo del periodista. Tauros, España.

Grillo, A. (2001), Días extraños, cubrimientos extraños, Palabra Clave, Universidad de La Sabana, Bogotá Colombia, número 005, Red de revistas científicas de América Latina y El Caribe, España y Portugal.

Gutiérrez Vidrio, S. (2010), "Discurso periodístico: una propuesta analítica". Revista Comunicación y Sociedad, núm. 14, julio-diciembre, Pp. 169-198. Universidad de Guadalajara, México.

Martínez Alberto, J.L. (1988), La comunicación periodística frente al reto electrónico. El retorno a los géneros. Cuenta y Razón, 34, 59-64.

Martínez Alberto, J.L. (2002), Curso general de redacción periodística: Lenguaje, estilos y géneros periodísticos en prensa, radio, televisión y cine, Quinta edición, Thompson: Estados Unidos.

Martín Vivaldi, G. (1981), Géneros periodísticos. Paraninfo.

McLuhan, M. (1969), La comprensión de los medios como las extensiones del hombre. México: Diana.

Merlo, T. (2002), "La acción socializadora de la televisión en la época global". Revista Comunicar, 018, 35-40. Recuperado de: http://dialnet.unirioja.es/servlet/ articulo? codigo $=232456$

Santamaria (1990), El comentario periodístico: Los géneros persuasivos. Paraninfo, 1990.

Unicef (2005), Imagen de la infancia en los medios masivos de comunicación de Paraguay.

Unicef (2006), Convención de los derechos del niño.

Unicef (2007), Gasto público social para la infancia. El Salvador: Unicef.

Unicef (2011), Estado mundial de la infancia 2011: La adolescencia, una época de oportunidades. Estados Unidos: Unicef. 